\title{
Physiopathologie de I'insuffisance cardiaque chronique d'origine coronarienne
}

L'insuffisance cardiaque chronique qui fait suite à un infarctus du myocarde cicatrisé a une physiopathologie complexe. L'éjection systolique est en effet diminuée pour plusieurs raisons, dont certaines peuvent être assez spécifiques d'espèce animale : (1) importance de la perte de substance; (2) qualité de la restauration de la masse tissulaire par hypertrophie des zones saines ; (3) caractère à la fois concentrique et excentrique de l'hypertrophie; (4) dysfonctionnement diastolique dû à des modifications, non univoques, de la rigidité de la chambre ventriculaire. Qualitativement, le processus adaptationnel se traduit par un ralentissement de la vitesse maximale de raccourcissement dû, selon les espèces, à un changement d'expression des isogènes codant pour certaines protéines contractiles, ou à des modifications membranaires, ou bien aux deux. Ces changements de structure membranaire devraient conduire à réviser la conception de nouveaux médicaments inotropes.

\section{Bernard Swynghedauw}

\section{ADRESSE}

B. Swynghedauw : docteur en médecine, docteur ès sciences, directeur de recherche à l'Inserm, directeur de l'U.127 de l'Inserm. Inserm U.127, hôpital Lariboisière, 41, boulevard de

'insuffisance cardiaque (IC), se définit comme un état pathologique dans lequel une anomalie dans la fonction cardiaque est responsable de l'incapacité où se trouve le cœur de pomper le sang en quantité suffisante pour assurer les besoins métaboliques des tissus périphériques. Cette définition est celle de l'OMS (Organisation Mondiale de la Santé) et souligne un point capital : contrairement à une opinion répandue, l'IC se définit par ses conséquences périphériques. Le muscle cardiaque peut avoir des performances intrinsèques diminuées, mais une vasoconstriction périphérique peut, par exemple, compenser les insuffisances du muscle et prévenir, au moins pendant un temps, l'apparition de l'IC.

L'IC d'origine coronarienne fait suite à une occlusion complète ou incomplète d'une artère coronaire, occlusion qui est, dans la très grande majorité des cas, due à une thrombose sur plaque d'athérome généralement associée à une sclérose. Cet état peut survenir dans l'évolution de la maladie coronarienne de trois manières : (1) de façon aiguë, transitoire, au cours d'une crise d'angine de poitrine, traduction clinique 
d'une ischémie réversible secondaire à une occlusion incomplète, rendue symptomatique par un exercice physique ; (2) de façon aiguë, dramatique, au tout début d'un infarctus myocardique, traduction clinique d'une occlusion artérielle complète, irréversible en l'absence d'une thérapeutique spécifique. La nécrose tissulaire peut être à l'origine de la rupture d'un pilier mitral ou de la perforation du septum et ainsi aggraver le tableau; (3) enfin dans les suites d'un infarctus cicatrisé, après une période d'hypertrophie compensatrice sans IC [1, 2] ; c'est cette dernière éventualité qui sera détaillée ici, car c'est elle qui pose le plus de problèmes en physiopathologie. C'est aussi avec l'hypertension artérielle, l'une des deux grandes causes d'insuffisance cardiaque.

La survenue d'une IC est un mauvais élément du pronostic au cours de l'insuffisance coronarienne. Cette complication est, de ce fait, une des grandes causes de mortalité de la maladie athéroscléreuse.

Deux associations morbides sont particulièrement fréquentes et jouent un rôle très particulier dans la genèse de l'IC: (l) l'hypertension artérielle essentielle qui est en soi un facteur athérogène. C'est également, par la surcharge de pression qu'elle impose, une cause d'IC; (2) le diabète est également en soi un facteur favorisant le développement de l'athérome. La maladie diabétique a, par ailleurs et indépendamment de ce processus, une traduction cardiaque.

\section{La structure du myocarde dans I'hypertrophie et I'IC chronique d'origine coronarienne}

Après un délai qui va de un à plusieurs jours, la zone privée de sang va se nécroser, être envahie par des fibroblastes et se cicatriser. Parallèlement, le myocarde sain va s'hypertrophier compensant, au gramme près, la perte de substance, pourvu que celle-ci soit modérée (figure 1). Cette hypertrophie, comme toujours dans le myocarde adulte, est due à une hypertrophie des myocytes et de leur noyau, sans mitoses, le cardiocyte a perdu cette propriété [3]. Elle s'ac$\mathrm{m} / \mathrm{s} n^{\circ} 6$ vol. 5 , juin 89 compagne d'une hypertrophie et d'une multiplication des cellules non musculaires endothéliales, capillaires entre autres. Ce deuxième mécanisme compensateur est inadéquat et, sous l'endocarde, le myocarde sain hypertrophié est mal vascularisé, comme dans tous les autres types d'hypertrophie [4]. L'hypertrophie des myocytes a des limites : dans les infarctus étendus, la perte de substance n'est plus compensée au gramme près et la masse contractile totale est réduite.

L'hypertrophie revêt un double aspect, très spécifique de ce type d'étiologie: concentrique, le diamètre moyen des fibres augmente comme au cours d'une surcharge de pression (sténose aortique), et excentrique, la longueur des fibres et des myocytes augmente comme dans une surcharge de volume [5]. Ceci est le reflet d'une physiopathologie complexe: (1) l'hypertrophie concentrique compense l'augmentation des contraintes imposées à la paroi par la cicatrice; $(2)$. dès que la perte de substance survient, le myocarde compense le déficit en tissu contractile en utilisant les possibilités de la loi Starling ${ }^{*}$, c'est-à-dire en se dilatant et en élevant ses pressions télédiastoliques, tout comme dans une surcharge de volume. Ce type de mécanisme étire en permanence les myocytes, puisqu'au stade d'IC volume et pression diastoliques sont élevés (Tableau I)

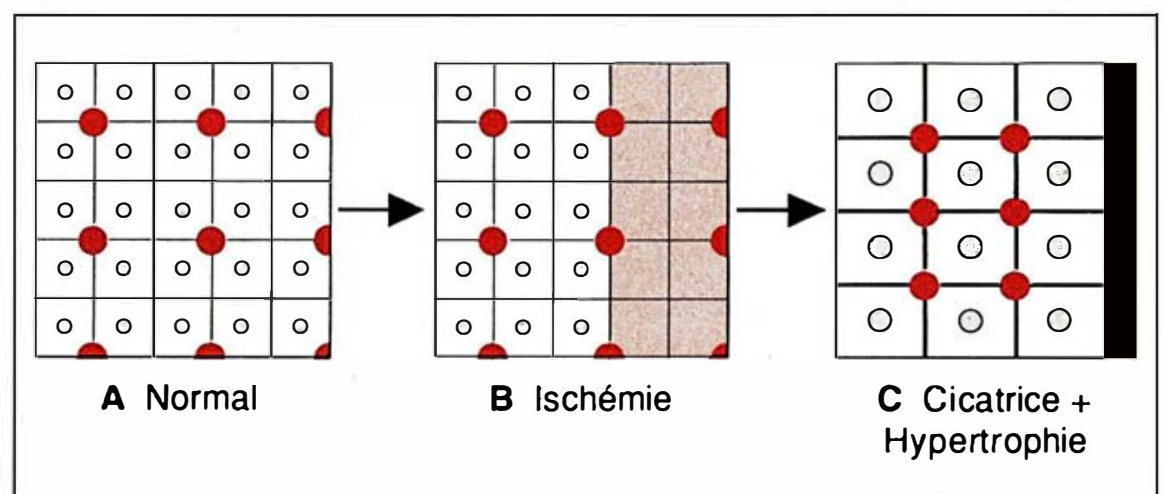

Figure 1. Hypertrophie cardiaque compensatrice au décours d'un infarctus du myocarde. On a schématisé chez un rat normal (A) les myocytes $\left(753 / \mathrm{mm}^{2}\right.$ occupant un volume de $\left.673 \mathrm{~mm}^{3}\right)$, leurs noyaux, colorés en gris (diamètre moyen, Dn, 17,91 $\mu \mathrm{m}$ ) et la lumière des vaisseaux capillaires (cercles rouges) ( $\simeq 80 \mathrm{~mm}^{2} / \mathrm{mm}^{3}$ de myocytes). (B), Zone ischémiée en rose. (C) Infarctus limité, hypertrophie compensatrice et zone cicatricielle. Le poids du cœur (surface totale du carré) change peu, l'hypertrophie des myocytes compense la perte de substance. II y a moins de myocytes $\left(610^{*}\right)$, mais ils sont plus longs $\left(120 \mu m^{*}\right.$ au lieu de $100 \mu \mathrm{m})$ et plus épais $\left(220 \mu \mathrm{m}^{2}\right.$ à la section au lieu de $\left.200 \mu \mathrm{m}^{2}\right)$, néanrnoins ils occupent moins de place dans le myocarde (volume occupé $634 \mathrm{~mm}^{3}$ ). IIs possèdent des noyaux plus gros $\left(\mathrm{Dn}, 18,54^{*}\right)$. La croissance des capillaires est inadéquate $\left(\simeq 70 \mathrm{~mm}^{2}\right.$ de lumière capillaire par $\mathrm{mm}^{3}$ de myocytes $*$ ). La cicatrice d'infarctus est représentée par la bande noire verticale. * signifie que les différences sont, pour les auteurs, significatives [données d'après 5].

* Loi de Starling. Un cœur normal va éjecter à chaque systole une quantité de sang rigoureusement égale à celle qui y pénètre à la diastole. Cette loi n'est que la variation "cœur" de la courbe tension/longueur qui est une des trois caractéristiques physiologiques élémentaires d'un muscle (cardiaque ou squelettique). Cette courbe a une forme en cloche avec des fibres musculaires. Sur une sphère, comme le coeur, seule la partie ascendante de la courbe en cloche existe. Le volume en fin de diastole représente la longueur initiale, son augmentation va, par simple mise en jeu de la loi de Starling, augmenter la tension active développée. Biochimiquement, le sommet de la courbe en cloche, ou le haut de la courbe de Starling correspond au point où les ponts établis par la myosine avec l'actine sont les plus nombreux, et où donc la force développée sera la plus grande.

** Note correspondant à l'appel de la p. 399. Il est d'usage de distinguer ces deux types de surcharge mécanique ou chimique comme en expérimentation animale. La première se caractérise par un épaississement important de la paroi sans dilatation. Elle compense une surcharge de pression, hypertension artérielle par exemple, c'est-à-dire qu'elle survient lorsqu'il y a obstacle à l'éjection systolique. Dans l'hypertrophie excentrique, la dilatation prédomine, le poids de l'organe augmente mais surtout son volume et la paroi reste proche de la normale. Ceci se voit lorsque le coeur est obligé de travailler en permanence avec un volume télédiastolique élevé, dans une insuffisance aortique par exemple. Histologiquement, ces deux types de surcharge sont différents, à l'exception du collagène; les paramètres biochimiques sont, en revanche, comparables. 


\section{RÉFÉRENCES}

1. Bounhoure JP. Insuffisance cardiaque. Problèmes actuels. Paris: Baillières, 1988 : 333.

2. Fozzart HA, Haber $E$, Jennings RB, Katz AM, Morgan HE. The heart and cardiovascular system. Scientific foundations, 2 vol. New York : Raven Press, 1986 : 1694

3. Swynghedauw B, Delcayre C. Biology of cardiac overload. Pathobiol Ann 1982; 12: 137-83.

4. Rembert JC, Kleinman LH, Feder JM, Wechsler AS, Greenfield JC Jr. Myocardial blood flow distribution in concentric left ventricular hypertrophy. I Clin Invest 1978; 72 : 379-86.

5. Anversa P, Beghi C, Kikkawa Y, Olivetti G. Myocardial infarction in rats. Infarct size, myocyte hypertrophy, and capillary growth. Circ Res 1986 ; 58 : 26-37.

6. Gaasch WH, Apstein CS, Levine HJ. Diastolic properties of the left ventricle. In : Levine HJ, Gaasch WH, eds. The Ventricle: Basic and Clinical Aspects. Boston: Martin Nijhoff, 1985 : 143-70.

7. Apstein CS, Grossman W. Opposite initial effects of supply and demand ischemia on left ventricular diastolic compliance : the ischemia-diastolic paradox. J Mol Cell Cardiol $1987 ; 19: 119-28$

8. Pfeffer J, Pfeffer MA, Braunwald E. Hemodynamic benefits and prolonged survival with long-term captopril therapy in rats with myocardial infarction and heart failure. Circulation 1987 ; 75 : 149-55.

9. Weber KT, Janicki JS, Shroff SG, Prick R, Chen RM, Bashey RI. Collagen remodeling of the pressure-overloaded, hypertrophied non-human primate myocardium. Circ Res $1988 ; 62$ : 757-65.

10. Whittaker P, Boughner DR. Collagen organization in myocardial scars. Circulation 1988; 78 : 2134-8.

11. Lecarpentier Y, Waldenstrom A, Clergue $\mathbf{M}$, et al. Major alterations in relaxation during cardiac hypertrophy induced by aortic stenosis in guinea pig. Circ Res 1987; 61 : 107-16

12. Hanf R, Durubaix I, Lelièvre L. Rat cardiac hypertrophy : altered sodium-calcium exchange activity in sarcolemmal vesicles. FEBS Lett 1988 ; 236 : 145-9.

13. Gwathmey JK, Morgan JP. Altered calcium handling in experimental pressureoverload hypertrophy in the ferret. Circ Res $1985 ; 57: 836-43$.

14. Swynghedauw B. Developmental and functional adaptation of contractile proteins in cardiac and skeletal muscles. Physiol Rev
Ainsi, si la perte de substance contractile est bien entendu le premier des facteurs étiologiques de l'IC, les caractéristiques de la cicatrisation et de l'hypertrophie compensatrice vont interagir de façon complexe et aboutir à une IC à la fois d'origine systolique et d'origine diastolique.

\section{Le dysfonctionnement diastolique}

Le remplissage des cavités ventriculaires au moment de la diastole dépend bien évidemment de la vitesse à laquelle le muscle se relâche mais aussi et surtout de la rigidité de la chambre ventriculaire (ou de sa compliance, qui est à l'inverse de la rigidité). Les anomalies de la diastole sont précoces et précèdent en règle les changements dans l'éjection systolique.

La rigidité de la chambre ventriculaire. La rigidité de la chambre (chamber stiffness) se détermine en établissant en fin de diastole une courbe pression-volume. Les variations de ces paramètres sont obtenues in vivo en perfusant des solutions de macromolécules (figure $2 A$ ) et in vitro, sur cœur isolé, en gonflant le cœur avec du sérum physiologique par l'intermédiaire d'un ballonnet (figure $2 B$ ). Ce moment du cycle cardiaque est choisi car il reflète par définition l'étendue maximale de

la relaxation alors qu'il ne dépend pas de la vitesse de relaxation [6, 7]. Lorsque la courbe pression-volume diastolique (figure 2) est décalée vers la gauche, cela veut dire que, pour un même volume, la pression sera plus élevée et que donc la rigidité de la chambre est plus grande, ou sa compliance plus faible.

La rigidité de la chambre dépend elle-même de deux paramètres qui peuvent, selon l'étendue de la zone cicatricielle, évoluer en sens inverse : la rigidité du tissu (myocardial stiffness) et le volume de la cavité. Chez le chien, qui ne survit plusieurs semaines qu'à des infarctus cicatrisés de petite dimension, la rigidité du tissu joue un rôle majeur car la cavité ventriculaire se dilate peu ; la rigidité de la chambre y est normale ou aug. mentée. C'est l'inverse chez le rat, qui tolère des infarctus de grande dimension au prix d'une augmentation importante de son volume diastolique (figure $2 B$ ) et chez qui, après un infarctus, la rigidité de la chambre diminue [6].

La rigidité du tissu dépend essentiellement, mais probablement pas exclusivement, de sa composition, en termes quantitatifs et qualitatifs, en collagène [9]. Il faut considérer plusieurs éléments : la cicatrice ellemême, faite de collagène de type I [10]; sa périphérie, faite de collagène de type III ; la zone saine, hypertrophiée, plus riche en collagène que du

\begin{tabular}{|lcc|}
\hline \multicolumn{3}{|c|}{ Tableau I } \\
CARACTËRES ANATOMIQUES ET HÉMODYNAMIQUES \\
PRINCIPAUX DE L'INFARCTUS DU MYOCARDE EXPÉRIMENTAL \\
DU RAT $[5,8,22]$
\end{tabular}

${ }^{*}: p<0,05 ;{ }^{*}: p<0,01$. 


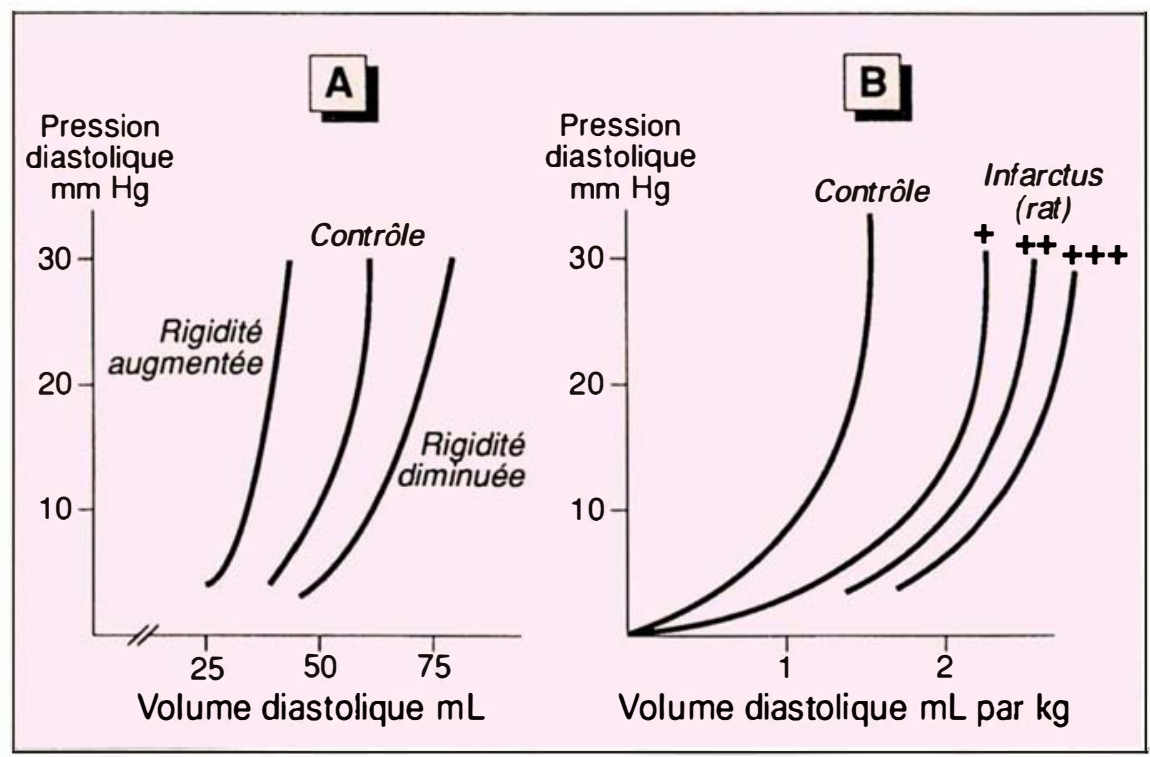

Figure 2. Mode de détermination de la rigidité de la chambre ventriculaire. Il consiste à établir au cours de la diastole une courbe pression-volume. La tangente à cette courbe représente la rigidité de la chambre pour une pression diastolique donnée, cette rigidité augmente linéairement avec la pression. (A) Courbe obtenue in vivo en perfusant une solution de macromolécules (d'après [6] et des données du laboratoire). (B) Courbe obtenue sur cœur isolé de rat témoin ou 8 semaines après ligature coronaire.,+++ et +++ indiquent la taille de la cicatrice myocardique. [D'après 22.]

tissu normal, faite de collagènes de types I et III entremêlés.

$\mathrm{La}$ concentration en collagène du myocarde est presque doublée (Tableau II), surtout sous l'endocarde. La nature mixte, excentrique et concentrique ** (voir note p. 397), de ce type d'hypertrophie permet de prédire, par analogie, l'existence à la fois d'un remaniement du réseau du collagène, comme dans les surcharges de pression, et la multiplication des ponts entre les fibres de collagène de type I aussi bien que de type II, bien connue dans les surcharges de volume. Les changements dans la structure du collagène précédant, dans les surcharges de pression, les modifications de la contractilité.

La relaxation du muscle cardiaque. Le retour à une tension et à une longueur présystoliques se fait in vivo en deux phases: la première, isovolumique, va de la fermeture des valves aortiques à l'ouverture des valves mitrales; la seconde est une phase de remplissage ventriculaire. La relaxation est un processus complexe qui dépend à la fois d'un phé$\mathrm{m} / \mathrm{s} n^{\circ} 6$ vol. 5 , juin 89 nomène actif de désactivation de l'appareil contractile et de l'état antérieur de la charge. L'analyse de ces paramètres n'est pas aisée en physiologie, la relaxation est ralentie dans ce modèle d'insuffisance cardiaque chronique d'origine coronarienne
[11], mais le détail en est mal connu. Au niveau du myocyte, la relaxation dépend du départ du calcium fixé sur l'appareil contractile; elle sousentend des phénomènes actifs, puisque la concentration cytosolique en calcium libre est plusieurs milliers de fois plus basse dans le cytoplasme qu'à l'extérieur ou que dans le réticulum sarcoplasmique. La cellule doit, pour se relaxer, abaisser la concentration en $\mathrm{Ca}^{2+}$ libre qui est, au pic de la contraction, autour de $10^{-6}-10^{-5} \mathrm{M}$. Elle utilise pour ce faire trois mécanismes : une $\mathrm{Ca}^{2+}$ ATPase qui concentre le $\mathrm{Ca}^{2}{ }^{+}$cytosolique dans une structure sacculaire intracellulaire, le réticulum sarcoplasmique; une autre ATPase qui expulse le cation hors de la cellule ; enfin, un système électrogénique d'échange $\mathrm{Na}^{+} / \mathrm{Ca}^{2+}$. L'importance relative de ces systèmes est spécifique d'une espèce et d'un type de muscle.

Deux de ces mécanismes ont été étudiés dans les hypertrophies concentriques et y sont modifiés, tout au moins chez le rat, chez qui on a démontré que la densité de la $\mathrm{Ca}^{2+}$ ATPase du réticulum sarcoplasmique est diminuée, ce qui explique que la capacité de cette structure intracellulaire à capter le $\mathrm{Ca}^{2+}$ est diminuée (données non publiées du laboratoire). Par ailleurs, in vitro, l'activité de l'échange $\mathrm{Na}^{+} / \mathrm{Ca}^{2+}$ est également diminuée, ce qui pourrait être dû également à une baisse de la densité en protéines échangeuses $\mathrm{Na}^{+} / \mathrm{Ca}^{2}{ }^{+}$. L'ensemble rend assez

\begin{tabular}{|c|c|c|}
\hline \multicolumn{3}{|c|}{$\begin{array}{c}\text { Tableau ॥ } \\
\text { CARACTĖRES BIOCHIMIQUES PRINCIPAUX } \\
\text { DE L'INFARCTUS DU MYOCARDE EXPÉRIMENTAL DU RAT }\end{array}$} \\
\hline & Témoins & Infarctus \\
\hline \multicolumn{3}{|l|}{$\begin{array}{l}\text { Densité en collagène du tissu non infarci } \\
\text { (en \%) [22] }\end{array}$} \\
\hline $\begin{array}{l}\text { sous l'endocarde } \\
\text { sous l'épicarde }\end{array}$ & $\begin{array}{l}2,68 \\
2,80\end{array}$ & $\begin{array}{l}4,14^{*} \\
3,50\end{array}$ \\
\hline \multicolumn{3}{|l|}{$\begin{array}{l}\text { Profil isoenzymatique de la myosine (en } \\
\% \text { ) [21] }\end{array}$} \\
\hline $\begin{array}{l}\text { V1 (homodimère } \alpha \alpha \text { à activité ATPase } \\
\text { élevée) }\end{array}$ & $90-100$ & $35^{*}$ \\
\hline $\begin{array}{l}\text { V3 (homodimère } \beta \beta \text { à activité ATPase } \\
\text { basse) }\end{array}$ & $0-100$ & $29 *$ \\
\hline
\end{tabular}

La densité en collagène est mesurée par morphométrie. Le pourcentage des isomyosines est mesuré après séparation électrophorétique $[21,22]{ }^{*}: p<0,01$ 
bien compte du fait que la relaxation active du cœur hypertrophié est, comme la contraction, ralentie [12, 13].

\section{La fonction systolique}

C'est bien évidemment d'elle que dépend directement l'irrigation des tissus, mais, dans ce type d'IC, les déterminants de l'éjection systolique sont plus complexes que dans une IC faisant suite à une simple surcharge de pression, secondaire à une hypertension artérielle par exemple. Ce domaine est une des sources majeures d'incompréhension entre les fondamentalistes et les cliniciens. Il y a en fait, dans le déterminisme de l'IC d'origine coronarienne, quatre groupes de paramètres intriqués : (1) des paramètres propres à cette cause : la perte de substance, qui peut jouer un rôle majeur si elle dépasse un certain seuil, la rigidité de la chambre ventriculaire qui, en gênant le remplissage, va forcément diminuer l'éjection, l'hétérogénéité segmentaire source d'asynchronisme, et les troubles du rythme; (2) certains mécanismes compensateurs périphériques de nature neuro-endocrinienne: la sécrétion d'angiotensineII, de catécholamines est stimulée par la baisse du débit cardiaque. Bénéfiques, ils peuvent permettre à l'organisme de continuer à irriguer les organes nobles aux dépens de la peau, par exemple. En revanche, ces hormones ont toutes des effets délétères sur le tissu cardiaque et, comme certains développements pharmacologiques récents l'ont montré, entraînent une suradaptation nuisible* (voir note p. 401) ; (3) l'hypertrophie cardiaque n'est pas que compensatrice; l'angiogenèse ne suit à l'évidence pas, et, comme nous l'avons vu plus haut, tous les cœurs hypertrophiés souffrent d'une vascularisation coronaire sous-endocardique sous-développée $[4,5]$; (4) les limites du processus d'adaptation. L'hypertrophie compensatrice, comme d'ailleurs tous les processus adaptatifs, à l'hypoxie par exemple, est loin d'être parfaite au moins sur deux points : (a) L'hypertrophie tissulaire est un processus d'adaptation à la fois parce qu'elle multiplie les unités contractiles pro- portionnellement à la perte de substance et parce qu'elle réduit la contrainte pariétale. Mais, on l'a vu, elle peut être insuffisante en cas de perte de substance étendue ; par ailleurs, elle modifie per se la rigidité de la chambre; (b) le ralentissement de la vitesse de raccourcissement constitue certes une adaptation au niveau de la fibre cardiaque ou d'une fibre musculaire squelettique [14], puisqu'il permet au muscle, au cours d'une surcharge mécanique, de maintenir sa tension active, aux dépens de la vitesse à laquelle elle se développe (figure 3). Au niveau de l'organe-cœur ceci aboutit néanmoins inéluctablement à une baisse du débit cardiaque (ou de l'index cardiaque, Tableau I) et donc à l'IC.

\section{Le processus biologique d'adaptation du cour}

Les modifications, évidentes, du phénotype dans le processus d'adaptation du cœur à une surcharge cardiaque sous-entendent l'existence d'une transduction mécanogénique, c'està-dire d'un processus par lequel le génome est informé des phénomènes mécaniques. La réponse du génome est double : (1) immédiate, transitoire sous la forme de signaux intracellulaires; (2) différée, permanente, adaptative. Elle est dans ce dernier cas à la fois quantitative (le myocyte s'hypertrophie) et qualitative (il y a changement d'expression de plusieurs isogènes). Le type de réponse qualitative est par ailleurs spécifique d'espèce, et n'est pas le même dans les ventricules et les oreillettes.

Les données physiologiques. La figure 3 résume le pourquoi et le comment du ralentissement de la vitesse maximale de raccourcissement, Vmax, phénomène adaptatif majeur, commun à tous les types de muscles [14]. Ce ralentissement est associé à un allongement de la durée du potentiel d'action, sans modification de la densité du courant entrant calcique étudié (patch-clamp); les modifications transitoires de la concentration intracellulaire en calcium est ralentie et la production de chaleur, dépendante ou non de la tension, est diminuée et retardée [3, 15 ]. 11. 


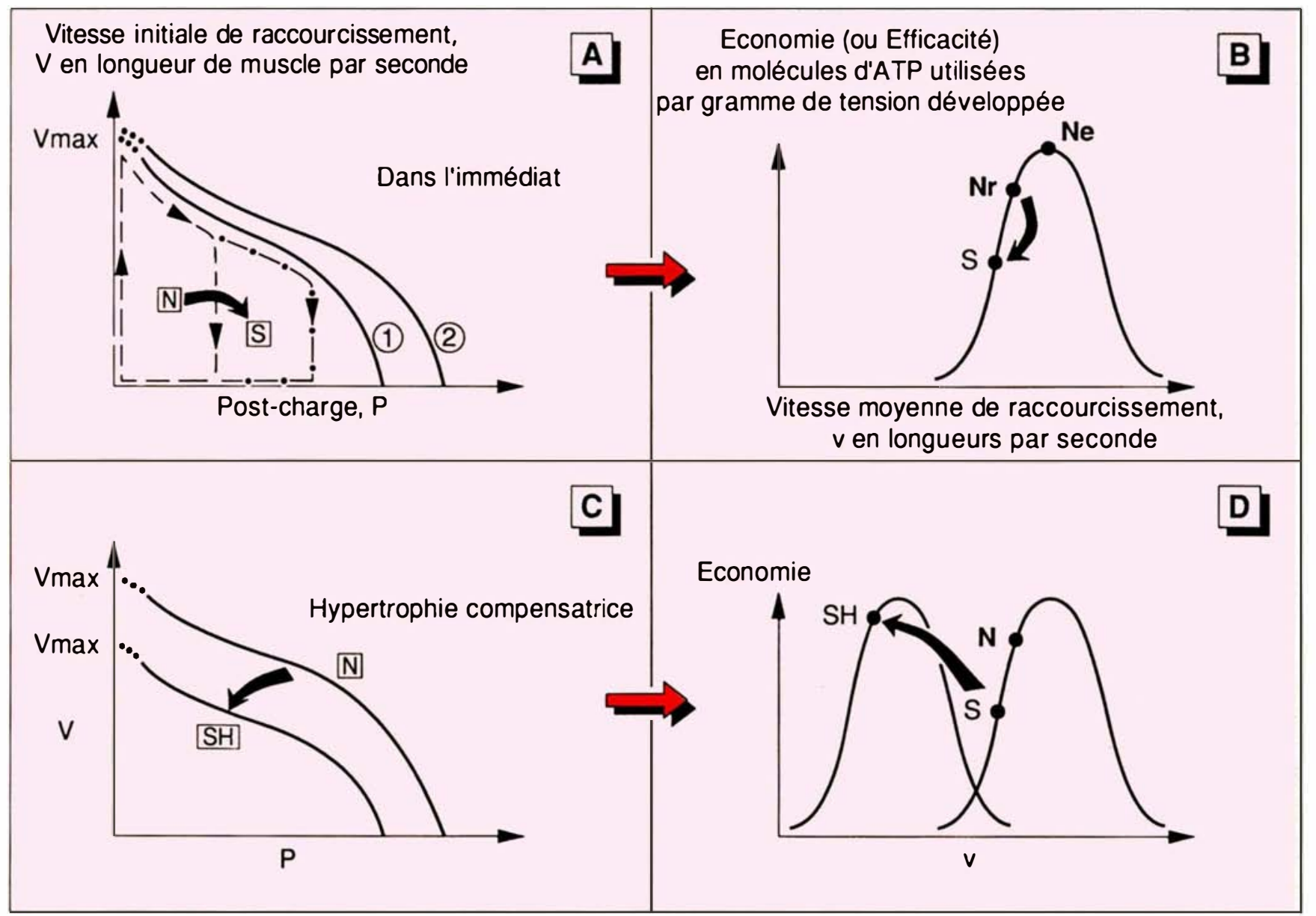

Les événements premiers. L'expression du génome cardiaque est modifiée sans intervention hormonale, ni intervention d'un médiateur de nature hormonale. Deux paramètres jouent, aussi bien sur myocyte isolé [19] que sur cœur isolé, un rôle indépendant : l'étirement cellulaire, paramètre également déterminant dans plusieurs tissus, et la tension isométrique. Le message qui informe le génome est inconnu, mais la première réponse, transitoire, du génome commence à être connue puisqu'on a démontré la rapide expression de deux oncogènes et d'au moins deux protéines de stress (ou heat-shock proteins) et l'existence d'un remaniement du cytosquelette [16-19].

La diminution de la Vmax. Elle peut s'expliquer soit par un changement dans les systèmes contrôlant les mouvements intracellulaires du calcium; soit par un changement des protéines contractiles - cible du calcium. Il
Figure 3. Mécanisme par lequel les changements dans la structure du myocarde permettent au cour de s'adapter à une surcharge de pression. (A) Événements immédiats. Courbe vitesse/post-charge réalisée sur fibre musculaire: à charge élevée la vitesse est nulle, à charge nulle la vitesse est maximale (Vmax); Vmax est indépendante de la longueur initiale à laquelle débute la contraction. En 1 et 2, deux longueurs initiales. Le cycle cardiaque in vivo figure en traits d'union, à l'état normal (N) ou en cas d'une surcharge mécanique (S). La surface indiquée est une puissance (c'est-à-dire un travail par une unité de temps). La puissance développée en $S$ est bien entendu plus forte qu'en $N$, mais la vitesse instantanée de raccourcissement est plus lente. (B) Conséquences thermodynamiques de la surcharge mécanique. La courbe économie/vitesse est en forme de cloche. Ne: cœur normal à l'exercice. Nr: cœur normal au repos. $S$ : surcharge mécanique, qui entraîne un effondrement de l'économie. (C) L'étirement, la tension, la puissance, le déséquilibre thermodynamique, vont modifier l'expression du génome cardiaque. Quantitativement, le cœur s'hypertrophie. Quantitativement, sa propriété intrinsèque la plus caractéristique, Vmax, change: Vmax diminue. La courbe vitesse/charge est décalée vers le bas au stade de surcharge mécanique chronique avec hypertrophie. (D) Conséquences thermodynamiques de ce changement d'expression du génome; le myocarde utilise une autre courbe économie/vitesse qui lui permet de maintenir un bon équilibre thermodynamique à vitesse plus lente.

* Note correspondant à l'appel de la p. 400. On peut en effet inhiber les effets des catécholamines par des $\beta$-antagonistes (partiels dans l'IC) et surtout abaisser les taux d'angiotensine II par les inhibiteurs de l'enzyme de conversion (IEC). Le traitement de l'IC par les IEC améliore de façon spectaculaire l'IC, l'étude Consensus a en effet montré pour la première fois dans l'histoire de la pharmacologie clinique que ces médicaments amélioraient non seulement la qualité de la vie mais aussi le taux de mortalité dans cette maladie. Expérimentalement, les effets des IEC sur la rigidité du coeur dans l'IC d'origine coranienne sont tout aussi spectaculaires [8, 22]. 
Tableau III

LES DÉTERMINANTS DE L'ÉJECTION SYSTOLIOUE DU CCEUR DANS L'INSUFFISANCE CARDIAQUE CHRONIQUE D'ORIGINE CORONARIENNE

1. L'importance de la perte de substance contractile

2. L'importance de l'hypertrophie compensatrice

3. La rigidité de la chambre ventriculaire fonction de :

- la rigidité de la zone infarcie

- la rigidité de la zone hypertrophiée

- et du rapport volume diastolique/masse ventriculaire

4. Les performances contractiles du myocarde non ischémié qui dépend elle-même :

- ralentissement de Vmax, mécanisme compensateur au niveau de la fibre

- des modifications de la charge dues à la rigidité de la zone infarcie

- de l'hétérogénéité segmentaire source d'asynchronisme

- et des troubles du rythme

est hautement probable que les deux mécanismes interviennent. Le changement des protéines contractiles porte sur les isomyosines et se fait au profit des formes à activité ATPasique basse (Tableau II). Il est transcriptionnel et n'intervient pas dans le ventricule de certaines espèces, comme l'homme, alors qu'il est déterminant dans l'oreillette dans toutes les espèces ou dans le ventricule de rat [14, 19-21]. Chez le cobaye, par exemple, la diminution adaptative de la Vmax persiste en l'absence de structure membranaire sur fibre cardiaque pelée [11], c'est-à-dire dépourvue de toute structure membranaire.

La membrane du cœur hypertrophié est modifiée. Bien qu'aucune étude systématique n'ait été conduite dans l'infarctus, de nombreuses données, y compris des données cliniques, permettent d'extrapoler à l'infarctus ce qui a été trouvé dans les surcharges de pression. Le myocyte s'hypertrophie sans se diviser, sa capacité membranaire, par exemple, passe de 133 à $271 \mathrm{pF}$ (picofarad) pour une hypertrophie de $60 \%$. Sa membrane croît en proportion, mais beaucoup plus au niveau des tubules $T^{*}$ qu'à celui de la surface elle-même. Schématiquement, on peut distinguer dans les protéines membranaires deux groupes: (1) celles dont la densité diminue, ce qui veut dire que le nombre de molécules par myocyte ne change pas: on peut supposer que les gènes codant pour ces protéines sont insensibles au processus d'adaptation. $\mathrm{La} \mathrm{Ca}^{2}+\mathrm{ATPase}$ du réticulum sarcoplasmique, les récepteurs
Bl adrénergiques et muscariniques, une des isoformes de la $\mathrm{Na}^{+}-\mathrm{K}^{+}$ ATPase, font partie de cette famille [23-26]; (2) celles au contraire dont la densité augmente ou reste inchangée. Dans ce groupe, le nombre de molécules par myocyte augmente, même si la densité ne change pas, puisque la cellule s'hypertrophie sans se diviser. Ceci suppose un génome sensible à la transduction mécanogénique. Les canaux calciques du sarcolemme, une autre isoforme de la $\mathrm{Na}^{+}-\mathrm{K}^{+}$ATPase font partie de ce groupe [25-27].

L'une des conséquences les plus importantes de ce type de modulation est que la quasi-totalité des « récepteurs » (bien que l'on puisse considérer stricto sensu la $\mathrm{Na}^{+1}-\mathrm{K}^{+}$ ATPase, qui est spécifiquement inhibée par un inotrope, l'ouabaïne, comme un récepteur) des drogues inotropes, chargées de rétablir la contractilité dans l'IC, sont modifiés. La serrure ayant changé, il est évident que la recherche industrielle de nouveaux médicaments inotropes, c'est-à-dire d'une clef adaptée à ce type de serrure, se doit d'en tenir compte, ce qui n'est actuellement pas le cas

\section{* Invaginations de la membrane externe d'un myocyte qui se produisent en regard des lignes $Z$ du sarcomère et facilitent l'accès intracellu- laire du calcium.}

\section{TIRÉS A PART}

B. Swynghedauw.
RÉFÉRENCES

24. Chevalier B, Mansier P, Callens-El Amrani F, Swynghedauw B. The beta adrenergic system is modified in compensatory pressure cardiac overload in rats : physiological and biochemical evidence. J Cardiovasc Pharmacol (sous presse).

25. Lelièvre LG, Maixent JM, Lorente $P$, Mouas C, Charlemagne D, Swynghedauw B. Prolonged responsiveness to ouabain in hypertrophied rat heart : physiogical and biochemical evidence. Am J Physiol 1986; 250 : H293-31.

26. Charlemagne D, Maixent JM, Preteseille $\mathrm{M}$, Lelièyre $\mathrm{L}$. Ouabain binding sites and $\left(\mathrm{Na}^{+}, \mathrm{K}^{+}\right)$-ATPase activity in rat cardiac hypertrophy. Expression of the neonatal forms. J Biol Chem 1986 ; 261 : 185-204.

27. Mayoux E, Callens F, Swynghedauw B, Charlemagne D. Adaptional process of the cardiac $\mathrm{Ca}^{2}+$ channels to pressure overload: biochemical and physiological properties of the dihydropyridine receptors in normal and hypertrophicd rat hearts. J Cardiovasc Pharmacol 1988 ; 12 : 390-6.

\section{Summary}

Pathophysiology of chronic heart failure due to coronary heart disease

Chronic heart failure following scared myocardial infarction has a rather complex origin. Ejection fraction is indeed lowered for several reasons, including the size of the myocardial deficit, the degree of compensatory hypertrophy, the excentric and concentric character of the hypertrophy, the diastolic dysfunction which is due to changes in chamber stiffness. Some are species-specific. From a qualitative point of view the adaptational process results in a slowing of the maximal shortening velocity which is due to a shift in isogene expression. This shift concerns contractile or membrane proteins, or both, depending of the animal species. The latter, i.e. the changes in membrane composition, may have considerable consequences in pharmacological research. 